\title{
Odometer Lever Arm Effect Compensation on Strapdown Inertia Navigation System Precise Alignment with Odometer
}

\author{
Zhi-yuan $\mathrm{Ma}^{1,}$, Zhi-yong Shi ${ }^{1, b}$ \\ ${ }^{1}$ School of Ordnance Engineering College, Shijiazhuang 050003,China. \\ amatgzyyx@163.com bhaikiluna@163.com
}

Keywords: strapdown inertia navigation system, odometer, initial alignment, lever arm effect

\begin{abstract}
Odometer lever arm effect on strapdown inertia navigation system initial alignment with odometer is studied in this paper. This paper presents an error compensation method of odometer lever arm. In this method, the difference between output velocity by odometer and velocity output by SINS is taken as a observed quantity and precise alignment was achieved through Kalman filter. The simulations shows that the method given by this paper can receive a higher alignment accuracy.
\end{abstract}

\section{Introduction}

The strapdown inertial navigation system (SINS) have important application in modern weapons. many weapon systems are installed the SINS. the performance of the SINS have important effects on weapon performance. The SINS initial alignment for a SINS is very important, alignment accuracy determines the performance of the strapdown inertial navigation system. SINS initial alignment in moving base became the focus of the science. Many scholars did a lot of study on GPS alignment and moving base transfer alignment. Moving base transfer alignment needs an expensive high-precision main SINS as a reference. GPS alignment is not anti-jamming. The odometer alignment has the advantages of low cost, completely independent. Related research are taken seriously.

At present, there are many research about SINS initial alignment ${ }^{[1-4]}$. Most of the literature have neglected the lever arm effects on initial alignment. In fact, there are three lever arms in SINS initial alignment by odometer, Odometer lever arm, SINS lever arm and internal lever $\mathrm{arm}^{[5]}$. Odometer lever arm refers to lever arm from odometer to vehicle's center of mass. Usually, the odometer was fixed in vehicle's front wheel. In wheeling, the speed of the odometer and the vehicle's center of mass is not the same. It will produce an adverse effect on the alignment accuracy if odometer lever arm ignored.

In this paper, the odometer output is compensated by the lever arm, and then the vehicle mass center speed can be measured by the odometer output. The difference between the speed of odometer measurements and the speed of SINS measurements was observed quantity. Inertial alignment was achieved through Kalman filter. The method does not need to add additional hardware and improved by algorithm.

\section{Derivation of odometer lever arm}

As shown in figure 1, it is an assumption that the origin of navigation frame is $O_{n}$. the origin of body frame $O_{b}$ is in the center of mass. the SINS was installed in the origin of body frame. odometer was installed at A point in space. The vector distance between body's center of mass and $O_{n}$ is $\mathbf{R}_{b}$. The vector distance between odometer installation location and $O_{n}$ is $\mathbf{R}_{A}$. The vector distance between body's center of mass and odometer installation is $\mathbf{r}$. according to vector addition relationship:

$$
\mathbf{R}_{A}=\mathbf{R}_{b}+\mathbf{r}
$$




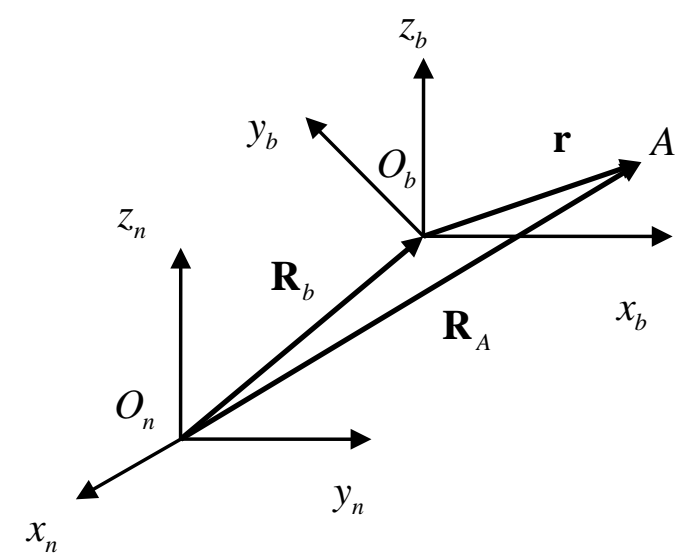

Fig. 1 Principle of lever arm effect

According to Coriolis theorem

$\frac{d_{n} \mathbf{R}_{A}}{d t}=\frac{d_{n} \mathbf{R}_{b}}{d t}+\frac{d_{n} \mathbf{r}}{d t}=\frac{d_{n} \mathbf{R}_{b}}{d t}+\frac{d_{b} \mathbf{r}}{d t}+\boldsymbol{\omega}_{n b}^{b} \times \mathbf{r}$

Body mass center is static. SINS and body frame is linked. So there is

$$
\begin{aligned}
& \frac{d_{n} \mathbf{R}_{b}}{d t}=0 \\
& \frac{d_{b} \mathbf{r}}{d t}=0
\end{aligned}
$$

The lever arm speed is given as

$$
\mathbf{v}_{L}^{b}=\frac{d_{n} \mathbf{R}_{A}}{d t}=\boldsymbol{\omega}_{n b}^{b} \times \mathbf{r}
$$

The speed relationship of SINS and odometer is given as

$$
\mathbf{v}_{\text {OD }}=\mathbf{v}_{\text {SINS }}+\mathbf{v}_{L}^{b}=\mathbf{v}_{\text {SINS }}+\boldsymbol{\omega}_{n b}^{b} \times \mathbf{r}
$$

The formula above is the compensation formula about odometer' the lever arm. In the formula, $\boldsymbol{\omega}_{n b}^{b}$ means rotating angular velocity from body frame to navigation frame projected on body frame. Its value is

$$
\boldsymbol{\omega}_{n b}^{b}=\boldsymbol{\omega}_{i b}^{b}-C_{n}^{b}\left(\boldsymbol{\omega}_{i e}^{n}+\boldsymbol{\omega}_{e n}^{n}\right)
$$

In the formula, $\boldsymbol{\omega}_{i b}^{b}$ means rotating angular velocity from body frame to inertial frame projected on body frame. Its values measured by the gyroscope. $\boldsymbol{\omega}_{i e}^{n}$ means rotating angular velocity from earth frame to inertial frame projected on navigation frame. Its value is

$$
\boldsymbol{\omega}_{i e}^{n}=\left[\begin{array}{lll}
0 & \omega_{i e} \cos L & \omega_{i e} \sin L
\end{array}\right]^{T}
$$

$\boldsymbol{\omega}_{e n}^{n}$ means rotating angular velocity from navigation frame to earth frame projected on navigation frame. Its value is

$$
\boldsymbol{\omega}_{e n}^{n}=\left[-\frac{v_{N}^{n}}{R_{M}} \quad \frac{v_{E}^{n}}{R_{N}} \quad \frac{v_{E}^{n}}{R_{N}} \tan L\right]^{T}
$$

In the formula above, $L$ means latitude, $\omega_{i e}$ means earth rotation angular velocity, $v_{N}^{n}$ means body north speed, $v_{E}^{n}$ means body east speed, $R_{M}$ means the earth curvature radius of meridian plane, $R_{N}$ means the earth curvature radius of prime vertical plane.

Through formula (6), the odometer measurements can be revised before filtering. Odometer lever arm error is eliminated and alignment accuracy is improved. 


\section{Filtering model}

The SINS/odometer integrated navigation coordinate system state equation on navigation frame is expressed as

$$
\mathbf{H}(\mathrm{t})=\mathbf{F}(\mathrm{t}) \mathbf{X}(\mathrm{t})+\mathbf{W}(\mathrm{t})
$$

The system state vector is taken as

$$
\mathbf{X}(\mathrm{t})=\left[\begin{array}{llll}
\boldsymbol{\varphi}^{T} & \delta \mathbf{v}^{T} & \boldsymbol{\varepsilon}^{T} & \nabla^{T}
\end{array}\right]^{T}
$$

In the formula above, $\boldsymbol{\varphi}^{T}$ means misalignment Angle, $\delta \mathbf{v}^{T}$ means the velocity error in the east and north , $\boldsymbol{\varepsilon}^{T}$ means gyroscope zero bias in three directions, $\nabla^{T}$ means accelerometers zero bias for the $\mathrm{x}$ and $\mathrm{y}$. $\mathbf{W}(\mathrm{t})$ means system state noise. Matrix value can be found in the literature [4].

The observation equation is taken as

$$
\mathbf{Z}(\mathrm{t})=\mathbf{H}(\mathrm{t}) \mathbf{X}(\mathrm{t})+\boldsymbol{\Theta}(\mathrm{t})
$$

It assumed that body speed in navigation frame calculated by SINS is $\mathbf{v}_{\text {SINS }}^{n}$, body speed in navigation frame calculated by odometer is $\mathbf{v}_{O D}^{n}$. velocity observation vector is defined as

$$
\mathbf{Z}(\mathrm{t})=\left[\mathbf{v}_{\text {SINS }}^{n}-\mathbf{v}_{O D}^{n}\right]=\left[\begin{array}{l}
v_{E, S I N S}^{n}-v_{E, O D}^{n} \\
v_{\mathrm{N}, S I N S}^{n}-v_{\mathrm{N}, O D}^{n}
\end{array}\right]
$$

It is launched as

$$
\mathbf{Z}(\mathrm{t})=\left[\begin{array}{l}
\delta v_{E} \\
\delta v_{N}
\end{array}\right]+\left[\begin{array}{ccc}
0 & v_{U, O D}^{n} & -v_{N, O D}^{n} \\
-v_{\mathrm{U}, O D}^{n} & 0 & v_{E, O D}^{n}
\end{array}\right]\left[\begin{array}{l}
\delta \alpha \\
\delta \beta \\
\delta \gamma
\end{array}\right]
$$

It can be calculated $\mathbf{H}(\mathrm{t})$ through formula (14).

\section{Simulation analysis}

It assumed that initial latitude is $30^{\circ}$ north latitude, initial longitude is $120^{\circ}$ east longitude, initial attitude Angle error is $0.1^{\circ}$. gyro constant drift is $0.01\left(^{\circ}\right) / h$, gyro white noise standard deviation is $0.001\left(^{\circ}\right) / \sqrt{h}$, accelerometer constant drift is $10^{-4} \mathrm{~g}$, accelerometer white noise standard deviation is $10^{-5} \mathrm{~g} g \sqrt{\mathrm{s}}$. Odometer is installed in the vehicle right front wheel. The lever arm has been obtained through calibration. The lever arm is taken as $\mathbf{r}_{O D}=\left[\begin{array}{lll}3 & 4 & -1\end{array}\right]^{T}$. Initial alignment was achieved through classical Kalman filter. The simulation time step is 0.1 second. The simulation time is 15 minutes.
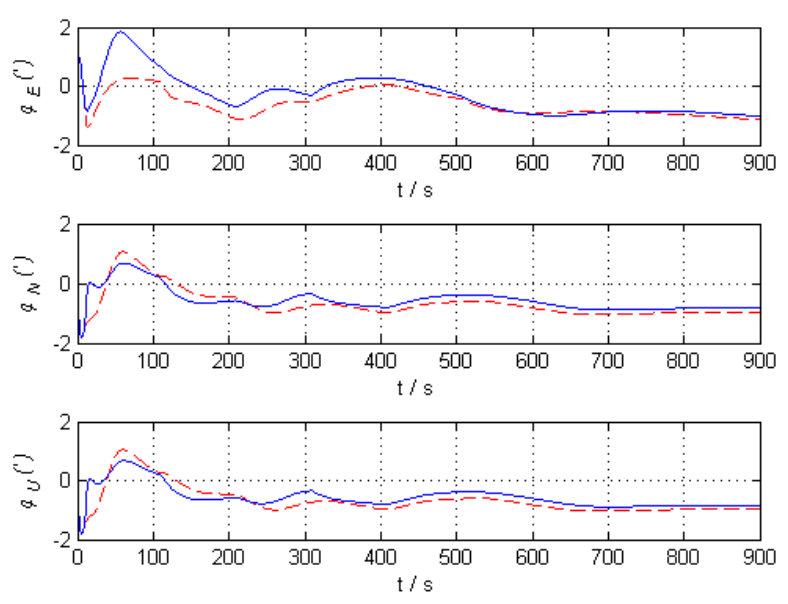

Fig. 2 Simulation of misalignment angles 
The misalignment Angle of simulation is shown in figure 2.The blue solid line means the misalignment angles had compensated the odometer lever arm. The red dotted line means the misalignment angles hadn't compensated the odometer lever arm. The simulation shows that the blue solid line is closer to straight line $\mathrm{y}=0$ than red dotted line. The lever arm compensation algorithm has a better convergence.

\section{Conclusion}

This paper proves that odometer lever arm error has an impact on SINS initial alignment through simulation, and put forward by odometer measurements through the lever arm compensation algorithm is correct, will be more close to the real value of odometer measurements into filtering calculation. By comparing the simulation verified the correctness of the algorithm

\section{References}

[1] Zhao Hong-song, Miao Ling-juan, Shen Jun, Zhang Xi. Research on Initial Alignment of SINS on Swaying Base through Velocity Measurement in Different Coordinates [J]. Journal of Armaments Factories, 2014, 35 (12) : 1951-1958.

[2] Yang Bo, Peng Pei-lin, Wang Yue-gang, Zhou xiao-gang. Alignment Method of Strapdown Inertial Navigation System Aided by Odometer on Moving Base [J]. Journal of Chinese Inertial Technology, 2013, 21 (3) : 298-307.

[3] Xiao Xuan, Wang Qing-zhe, Cheng Yuan, Fu Meng-yin, Liu Tony. High Accuracy Navigation Algorithm for Tightly Coupled INS/Odometer[J]. Journal of Chinese Inertial Technology, 2012, 20 (2) : 140-145.

[4] Chen Hong-yue, Sun Qian, Liu aerospace. SINS Alignment Algorithm for Marching Vehicles with the Aid of Distance-Transfer-Unit (DTU)[J]. Journal of Missile and Space Launch Technology, 2013, 5, 44 and 50.

[5] Yan Gong-min, Zhou Qi, Weng Jun, Qin Yong-yuan. Inner Lever Arm Compensation and Its Test Verification for SINS [J] Journal of Aerospace, 2012 (1) : 62-67. 